This is an Open Access article distributed under the terms of the Creative Commons Attribution-Noncommercial License, which permits unrestricted use, distribution, and reproduction in any noncommercial medium, provided the original work is properly cited.

\title{
MID-INFRARED DIRECT DETECTION OF EXTRA-SOLAR PLANETS
}

\author{
M. Fukagawa ${ }^{1}$, Y. Itoh ${ }^{2}$, and K. Enya ${ }^{3}$ \\ Department of Earth and Space Science, Graduate School of Science, Osaka University, 1-1 Machikaneyama, Toyonaka, \\ Osaka 560-0043 Japan \\ 32 Graduate School of Science, Kobe University, 1-1 Rokkodai, Nada, Kobe, Hyogo 657-8501, Japan \\ Research Institute of Science and Technology for Society, Japan Science and Technology Agency, Kawaguchi, Saitama
} $332-0012$

\section{ABSTRACT}

SPICA has the ability to directly detect Jovian planets in terms of sensitivity, and the additional coronagraphic capability will enable us to find not only free-floating but also wide companions with planetary masses. The spacebased telescope has an advantage in finding cooler planets around relatively old stars (>100 Myr - 5 Gyr), while ground-based surveys will find hotter, younger planets. We describe the observing mode and possible targets for SPICA coronagraphic observations of extra-solar planets. Low-resolution spectroscopy for known planets with $M_{p}>2$ $3 M_{J}$ may be interesting, while finding new cooler, older planets is also important.

Key words: Missions: SPICA - Stars: planetary systems

\section{INTRODUCTION}

The effort to find extra-solar planets and characterize them has been actively pursued in many ground-based projects and space-based missions. Detection of extra-solar planets is a rapidly growing field, and more than 400 planets have been found. Most of the known planets, however, were discovered by indirect methods such as radial velocity or transit surveys. The combination of these two methods gives the density of a planet, which is one of the important quantities to understand its nature. Spectroscopy for transiting planets has been used to investigate planets' atmospheres and hence another valuable tool to characterize them. While these methods are applicable only for highly inclined systems, direct imaging allows luminosity measurements or spectroscopy as follow-up studies for most of the planets.

SPICA has the ability to directly image planets in the mid-infrared regime. Figure 1 compares the spectra for Jupiter-mass objects of 1 Gyr at 10 pc (Burrows et al., 2003) and the sensitivity of SPICA. In addition, the coronagraphic capability will make it possible to detect not only free-floating planets but also planetary-mass companions orbiting a star like our solar system. The current design of the proposed coronagraph assumes an inner working angle of $\sim 3.3 \lambda / D$, which corresponds to $10 \mathrm{AU}$ for a $10 \mathrm{pc}$ star observed at $5 \mu \mathrm{m}$, and has slightly better contrast than JWST.
In this poster proceeding, we consider what kind of observations are to be pursued with SPICA.

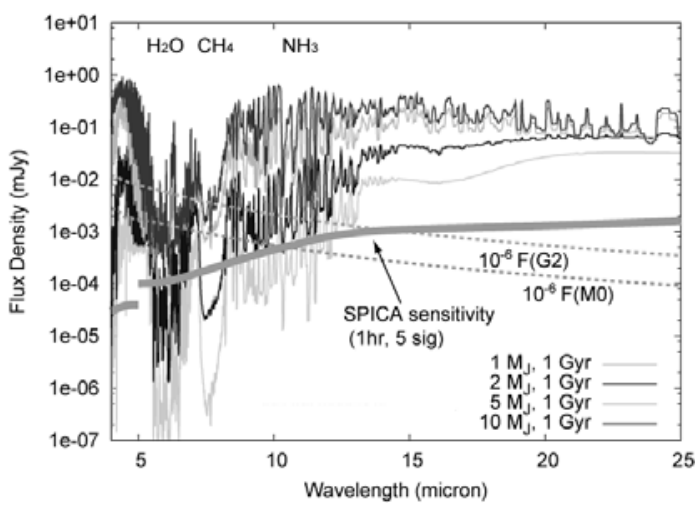

Figure 1. Model spectra for cool objects at 10 pc calculated by Burrows et al. (2003) overlaid with the SPICA sensitivity and the $10^{-6}$ flux levels for G2 and MO type star.

\section{IMAGing OR Spectroscopy}

Imaging observations will improve statistics on extra-solar giant planets at wide $\left(>1^{\prime \prime}\right)$ separations. Near-future projects using ground-based telescopes are planned, like GPI or SPHERE, and they will provide numerous new detections. However, ground-based surveys could suffer from high infrared background at around $5 \mu \mathrm{m}$ where planets are bright. In addition, detection of $\sim 1 M_{J}$ planets may be possible only for very young (Myr) stars due to the limited sensitivity. Therefore, space infrared telescopes will play a key role to find relatively old planets. On the other hand, JWST will also be a powerful telescope for planet search, but there may be an advantage for SPICA in finding planets at longer wavelengths where the high sensitivity will be achieved. In order to obtain good statistics, it may be useful to get short (minutes) exposures for a large number of targets, rather than to focus on deep imaging for lower-mass $\left(1 M_{J}\right)$ planets.

Spectroscopy for characterization of extra-solar giant planets is what we should consider given the significant science impact. The study of wide-separation planets is complementary to transit spectroscopy. Low-resolution spectroscopy is one possibility for planets that have been dis- 
covered with SPICA or other telescopes. Rich spectral features are expected at $<15 \mu \mathrm{m}$, such as $\mathrm{H}_{2} \mathrm{O}, \mathrm{CH}_{4}, \mathrm{NH}_{3}$, $\mathrm{PH}_{3}$, and $\mathrm{CH}_{3} \mathrm{D}$ seen in the spectra of Jupiter and Saturn (Encrenaz, 2008) (Figure 2), but we should keep in mind that there may be large variety of spectra for extrasolar planets depending on the weather as well as the planetary mass and location. For spectroscopy, sensitivity is an issue, but if we concentrate on the relatively massive planets $\left(>2-3 M_{J}\right)$, we can carry out low resolution $(R=$ several $\times 10)$ spectroscopy to detect band features.

Which wavelength is suited for the SPICA observations? The $5 \mu \mathrm{m}$ band is often recognized to be the good region for planet search as is obvious in Figure 1 and 2. In addition, closer planets will be able to be observed. The disadvantage at this short wavelength is the higher contrast between planets and the star, and this trend will be shown later in Figure 3. At wavelengths longer than $10 \mu \mathrm{m}$, planets are getting brighter, while stars are fainter. At $\lambda>15 \mu \mathrm{m}$, detectability is improved, thus suited for SPICA. However, since less spectral features are expected there (no obvious band absorption in Figure 1), it would not be wise to completely give up observing these bands even if the $5-15 \mu \mathrm{m}$ region can be observed also by JWST.

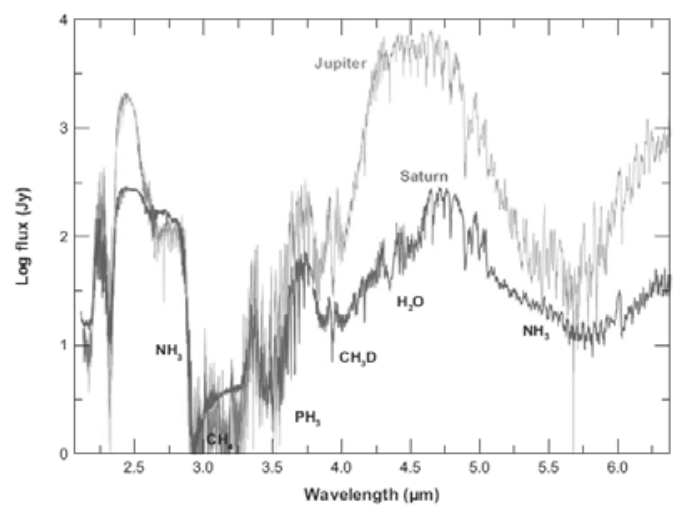

Figure 2. Spectra of Jupiter and Saturn obtained by ISO (Encrenaz, 2008).

\section{WHAT KINDS OF TARGETS ARE SUITED FOR SPICA?}

Here we summarize the pros and cons for pre-main-sequence stars (1-10 Myr), young main-sequence (0.1-1 Gyr), and relatively young Gyr stars.

\section{Forming Planets (1-10 Myr)}

pros. - Planets are brighter, and found at their birthplaces. Observations will be valuable to give constraints on theories of planet formation process.

cons. - Space observations are not necessarily required. Since nearby star-forming regions are relatively far, only outer planets ( $>100 \mathrm{AU})$ can be found.
Little advantage in discovery of planets since groundbased surveys will yield detections. SPICA will be able to measure the spectra of known planets in the midinfrared.

2. Young Planets (0.1-1 Gyr)

pros. - Planets are cooler than the younger ones, but space observations have enough sensitivity at longer wavelengths. Since planets almost forget their initial condition at these ages, a more robust discussion on the planetary mass might be possible compared to younger objects. In this age range, there are nearby moving groups or open clusters. Their ages are better determined than those of field stars.

cons. - Clusters and moving groups are already nominated as the targets for current and near-future planet surveys.

Although young planets are attractive as they are relatively bright and nearby, they are attractive also for other telescopes. It is advisable to avoid overlaps on targets with other projects. A search for lower-mass $\left(1 M_{J}\right)$ planets is possible, but it will need a large amount of time. Spectroscopy for nearby brighter members may be more interesting.

3. Planets around Young Population I stars (1-5 Gyr) pros. - Planets are cooler, thus space observations at long wavelengths have a clear advantage for these objects.

cons. - Only massive planets can be detected for older stars (>5 Gyr). A large uncertainty in stellar age is expected.

Spectroscopy for younger, brighter ones will be possible. It may also be interesting to search for outer planets in the planetary systems known by radial velocity measurements.

Figure 3 shows the signal-to-noise ratio (SNR) for $1 M_{J}$ planets with 1 hour integration against the inner working angle of the coronagraph. The contrast obtained by the coronagraph is assumed to be $10^{-6}$, and model spectra by Burrows et al. (1997) were used. Distant objects are fainter and have larger inner working angle if expressed in AU. The lower SNR is expected at shorter wavelengths even though the sensitivity is better, since the bright central star can be a source of noise. The first example is the Ursa Major group that is not too young (300-500 Myr), and a significant fraction of the member stars or possible members are very nearby (the average distance of the nucleus stars is $\sim 20$ pc) (King et al., 2003; Chupina et al., 2006). Each member candidate has not been extensively studied compared to other nearby groups perhaps due to the sparse distribution of stream stars, implying that our own work to establish a unique sample is still possible. In the second case, we consider nearby $(<25 \mathrm{pc})$ M type stars from the recent study by Shkolnik et al. (2009). High SNR are expected for such objects although 

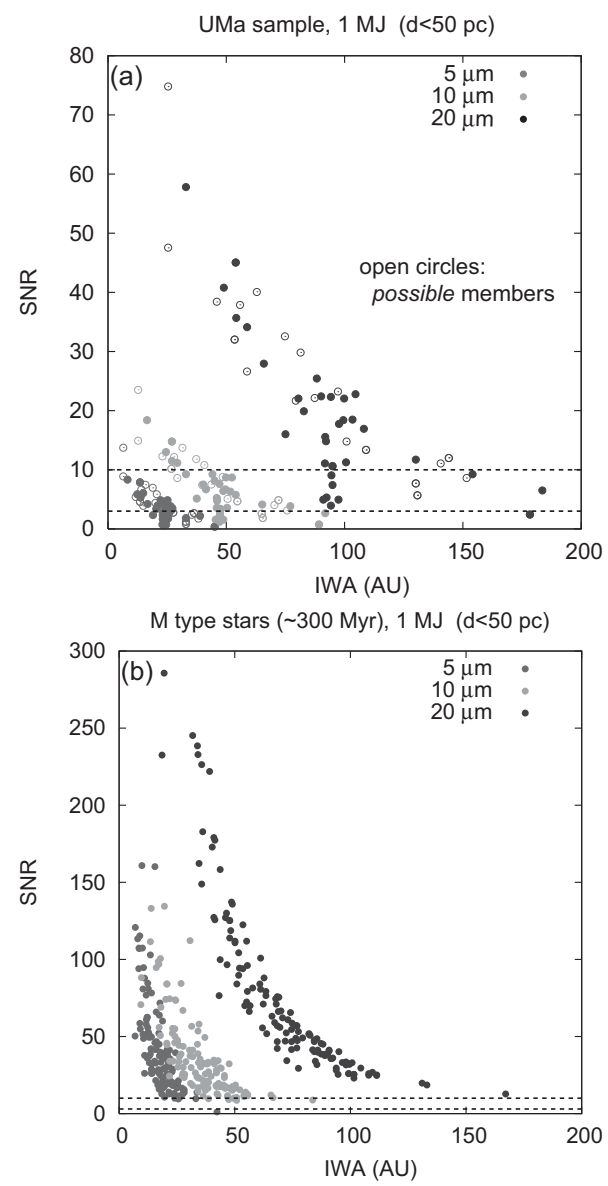

Figure 3. Signal-to-noise (1 hour observations) versus IWA in AU. (a) Nearby Ursa Major members by King et al. (2003). SNRs at short wavelengths are low since stars are very bright. (b) Young $M$ stars from Shkolnik et al. (2009). Advantage is in their proximity and faintness of the central stars.

the frequency for $\mathrm{M}$ type stars to have Jovian planets is observationally unclear. The estimate of SNR was also attempted for $1 \mathrm{Gyr}$ solar-type stars extracted from the NStED database, based on the ages derived from $R_{H K}^{\prime}$ and $L_{X}$ (Mamajek \& Hillenbrand, 2008). Three Jupitermass planets can be observed with SNR $>10$, with higher SNR at longer wavelengths.

\section{SUmmary}

Exploring extra-solar planetary atmospheres would become an important science case for SPICA. Finding new, cool, and wide-separation planets is also important, but spectroscopy for many detected planets even with low resolution (several tens) might be more productive and has a larger impact on this field. Spectroscopy with 1 hour integration might be useful to efficiently observe $M_{p}>2$ $3 M_{J}$ planets. It would be advisable to make spectroscopy from 5 to $20 \mu \mathrm{m}$, but 5 and $20 \mu \mathrm{m}$ bands may be enough for imaging observations to get closer and cooler planets. Short exposures (minutes) for a large number of targets are important to obtain statistics. A crude estimate suggests that it takes about 60 hours for 200 and 100 targets in the imaging mode in the age bin of $0.1-1 \mathrm{Gyr}$ and 15 Gyr, respectively, if we choose nearby targets $(<50 \mathrm{pc}$ for $0.1-1 \mathrm{Gyr},<25$ pc for $1-5 \mathrm{Gyr}$ ).

\section{REFERENCES}

Burrows, A., et al. 1997, ApJ, 491, 856

Burrows, A., Sudarsky, D., \& Lunine, J. I. 2003, ApJ, 596, 587

Chupina, N. V., Reva, V. G., \& Vereshchagin, S. V. 2006, A\&A, 451, 909

Encrenaz, T. 2008, ARA\&A, 46, 57

King, J. R., Villarreal, A. R., Soderblom, D. R., Gulliver, A. F., \& Adelman, S. J. 2003, AJ, 125, 1980

Mamajek, E. E., \& Hillenbrand, L. A. 2008, ApJ, 687, 1264

Shkolnik, E., Liu, M. C., \& Reid, I. N. 2009, ApJ, 699, 649 